\title{
VALIDITY AND APPLICABILITY OF TESTS FOR ORDERED ALTERNATIVES WITH BINARY RESPONSE FOR A CLINICAL DOSE-RESPONSE STUDY
}

\author{
Hiroyuki Uesaka
}

\begin{abstract}
This paper examines type I error rates and powers of several tests for ordered alternatives of a clinical dose-response study of a drug with dichotomous responses. The tests are analogues of tests for ordered alternatives for means of normal populations with known common variances. The tests are derived assuming asymptotic normality of estimates of response rates and are called large sample approximate tests. Type I error rates and powers of these tests are investigated through exact enumeration of the product binomial probabilities. The results show that Bartholomew's chi-bar test and Williams type test can be used in relatively small samples and also give high power for broad ordered alternatives.
\end{abstract}

\section{Introduction}

In clinical development of a new drug, a dose-response study is conducted to confirm existence of dose-response relationship. In general, efficacy rate of a drug increases with dose in a certain dose range of the drug and reaches to the maximum response. Some considers it to be important that the minimum effective dose and/or the maximum effective dose should be statistically identified. However, the minimum effective dose is usually defined as the lowest dose which shows statistically significant difference from the placebo. When a large placebo effect exists or when there are many non-responders, the difference of response rate between the lowest and highest doses may not be large enough to statistically identify the minimum effective dose in samples of moderate sizes.

In a dose response study of a drug on an efficacy variable, first of all it should be proved that there is statistically significant dose-dependent changes in the efficacy variable among several doses. When there are four or more dose levels, it is possible to estimate a dose-response curve by fitting a sigmoid curve and estimate the dose giving a specific amount of response which is clinically meaningful. Thus we are primarily interested in tests having high powers for broad ordered alternatives. Many authors have discussed tests against ordered alternatives in quantitative responses. Among them, Berenson (1982) and Shirley (1979),(1985) compared several tests with respect to powers and reported that, in general, Bartholomew's test and its nonparametric counterpart, which is proposed by Chacko (1963) and discussed by Shorack (1967), are recommendable. Budde and Bauer (1989) discuss multiple test procedures for dose-finding studies. Kuriki, Hirotsu and Hayter (1989) and Hirotsu, Kuriki and Hayter (1992) propose a test to find change point, which is referred to as the max-t test. Ruberg (1989) discusses use of contrasts to identify the minimum effective dose in animal experiments. As far as the author knows, there are only a few authors, Armitage (1955), Cochrane(1955) and Bartholomew (1959a), who discussed testing ordered alternatives for binomial populations. This is why the Cochran-Armitage test is so widely used in clinical trials in parallel group comparisons of several doses of a drug.

In the present paper, we develop several tests for ordered alternatives for binomial populations and examine their type I error rates and power properties of several tests against ordered alternatives.

Medical Statistics, Japan Clinical Research, Lilly Research Laboratories Japan, Eli Lilly Japan K.K. Sannomiya Plaza BLDG. 1-5, Isogamidori 7-chome, Chuo-ku, Kobe 651-0086, Japan 
The tests examined are analogues of tests for ordered alternatives for means of normal distributions with common known variances. The tests are derived from asymptotic normality of sample response rates, and the distribution of any test statistic examined is an unconditional approximate distribution derived from the asymptotic normal theory. The same test statistic is used to define conditional tests for the same alternative. Conditional tests are useful when sample sizes are small. In section 2 , several types of ordered alternatives are discussed. In section 3, tests against the ordered alternatives described in section 2 are discussed. Some of the tests are obtained by modifying tests derived for quantitative responses. In later sections, we examine type I error rates and powers of the tests by computing exact unconditional distributions. Before that in section 4 useful formulae for computing the distribution function of a test statistic are derived. Type I error rates and powers are examined in section 5 and 6 , respectively. In the final section 7 we illustrate sample size determination for a dose-response study.

\section{Situation and hypotheses}

Let $D_{1}, \cdots, D_{K}$ be $K$ doses of a drug to be tested, where $D_{1}<\cdots<D_{k}$. The lowest dose $D_{1}$ may be a placebo. Suppose $n$ subjects are randomly divided into $K$ subgroups and the $k$ th dose $D_{k}$ is administered to $n_{k}$ subjects of the $k$ th group. Let $r_{k}$ be the number of subjects who respond in the $k$ th group, $k=1, \cdots, K$ and let $r=r_{1}+\cdots+r_{K}$. The results are summarized in a $2 \times K$ contingency table. Let $p_{k}$ be the population response rate at dose $D_{k}$, and assume that $r_{k}$ has the binomial distribution with index $n_{k}, \quad k=1, \cdots, K$. For convenience, we write K-tuples of $p_{k} ' s, n_{k} ' s$ and $r_{k} ' s$ as $p=\left(p_{1}, \cdots, p_{k}\right), n=\left(n_{1}, \cdots n_{k}\right)$ and $\mathbf{r}=\left(\mathrm{r}_{1}, \cdots, \mathrm{r}_{\mathrm{K}}\right)$, respectively. If there is no drug effect,

$$
\mathrm{H}_{0}: \mathrm{p}_{1}=\cdots=\mathrm{p}_{\mathrm{K}}
$$

holds. Let us assume that the drug effect monotonically increases and this is represented as

$\mathrm{K}_{0}: \mathrm{p}_{1} \leq \cdot \mathrm{p}_{\mathrm{K}}$, where at least one of the inequalities is strict.

We further consider more restricted hypotheses as follows:

$$
\begin{aligned}
& \mathrm{K}_{1}: \mathrm{p}_{1}=\cdots=\mathrm{p}_{\mathrm{k}}<\mathrm{p}_{\mathrm{k}+1}=\cdots=\mathrm{p}_{\mathrm{K}} \text { for some } \mathrm{k} \quad(1 \leqq \mathrm{k}<\mathrm{K}-1), \\
& \mathrm{K}_{2}: \mathrm{p}_{1}=\cdots=\mathrm{p}_{\mathrm{k}}<\mathrm{p}_{\mathrm{k}+1} \leq \cdots \leq \mathrm{p}_{\mathrm{K}} \quad \text { for some } \mathrm{k} \quad(1 \leqq \mathrm{k}<\mathrm{K}-1),
\end{aligned}
$$

where $K_{1}$ is an analogue of a hypothesis discussed for normal means by Kuriki, Hirotsu and Hayter (1989), and $K_{2}$ is implicitly considered by Williams (1972). Next, let us consider a model $p_{k}=\alpha+\beta \theta\left(D_{k}\right)$, $\mathbf{k}=1, \cdots, \mathbf{K}$ where $\alpha$ and $\beta$ are unknown constants and $\theta(\mathbf{x})$ is a monotonically increasing function of $\mathbf{x}>0$. Then $\mathrm{H}_{0}$ implies that $\beta=0$ and an alternative $\mathrm{K}_{3}$ is defined by $\beta>0$. This hypothesis is implied by Cochran-Armitage test. Let us consider further a hypothesis

$$
\mathrm{K}_{4}: \mathrm{p}_{1}<\mathrm{p}_{\mathrm{k}} \text { at least one } \mathrm{k} \quad(1<\mathrm{k} \leqq \mathrm{K}) \text {. }
$$

Note that $\mathrm{K}_{4}$ neither is implied by $\mathrm{K}_{0}$ nor implies $\mathrm{K}_{0}$. Therefore this is not a dose-response hypothesis.

Let us denote the observed response rate $r_{k} / n_{k}$ by $R_{k}, k=1, \cdots, K, \quad R=\left(R_{1}, \ldots, R_{k}\right)$ and $R=r / n$. We call $R$ the raw estimate of $p$. The sums $r_{u}+\cdots+r_{v}$ and $n_{u}+\cdots+n_{v}$ are denoted by $r_{[u, v]}$ and $n_{[u, v]}$, respectively, for $1 \leqq \mathrm{u} \leqq \mathrm{v} \leqq \mathrm{K}$. An estimate of $\mathrm{p}_{\mathrm{k}}$ under $\mathrm{K}_{0}$ is given by well-known amalgamation procedure described by Bartholomew (1959a) and Barlow et al. (1972). The formula is

$$
R_{k}^{\cdot}=\max _{1 \leq u \leq k} \min _{k \leq v \leq \mathbf{K}} r_{[u, v]} / n_{[u, v]}, k=1, \cdots, K
$$

We call $\mathbf{R}^{*}=\left(\mathbf{R}_{1}^{*}, \cdots, \mathbf{R}_{\mathrm{K}}^{*}\right)$ max-min estimate of $\mathbf{p}=\left(\mathrm{p}_{1}, \cdots, \mathrm{p}_{\mathrm{K}}\right)$ under $\mathrm{K}_{0}$.

\section{Tests for ordered alternatives of binomial populations}

In this section, we briefly define statistical tests for testing ordered alternatives described in section 2 . Some of these tests are originally proposed for testing ordered alternative of mean values of normal populations with common known variances. When we apply these tests to dichotomous variables, we assume that the response variable $R_{k}$ has a binomial distribution with index $n_{k}$ and 
response probability $p_{k}, k=1, \cdots, K$. Then from the asymptotic normality of $R_{k}, k=1, \cdots, K$, any test statistics for testing $\mathrm{H}_{0}$ against $\mathrm{K}^{*}$, which will be discussed below, asymptotically has the distribution function of the corresponding test statistic defined for normal populations. Thus any test can be defined by using a critical point for the corresponding normal theory test. We will refer to these tests as large sample approximate tests in the subsequent discussion.

On the other hand, it is easy to define a test conditional on the observed total number of response $R$. This type of test which is defined by the same test statistic as the large sample approximate test can be performed by calculating exact conditional $p$ values using multivariate hypergeometric distribution. When sample sizes are small, the exact approach may be applied. Conditional tests are briefly discussed in a later section. However, in the subsequent sections, we examine only large sample approximate tests.

\subsection{Bartholomew's chi-bar square test}

Bartholomew (1959a) proposed a likelihood ratio test of means of normal populations under order restriction. He gave examples of testing $\mathrm{H}_{0}$ against $\mathrm{K}_{0}$ for binomial populations. The test statistic is

$$
\bar{\chi}^{2}=\sum_{k=1}^{K} n_{k}\left(R_{k}^{*}-R\right)^{2} / R(1-R) .
$$

Large sample approximate test of significance level $\alpha$ of $\mathrm{H}_{0}$ against $\mathrm{K}_{0}$ is defined as "reject $\mathrm{H}_{0}$ when $\bar{\chi}^{2} \geq \bar{\chi}^{2}(\alpha ; \mathrm{K})$, where $\bar{\chi}^{2}(\alpha ; \mathrm{K})$ is the upper $\alpha$ point of $\bar{\chi}^{2}$ statistic of Bartholomew (1959a). The distribution function of this statistic is given by Bartholomew(1959a,b) and Barlow et al. (1972). When $\mathrm{K}$ is 3 or 4 , the approximate null distribution function can be exactly calculated.

\subsection{Jonckheere type test}

Jonckheere (1954) and Terpstra (1954) proposed a distribution free test of homogeneity against the ordered alternative $\mathrm{K}_{0}$ for continuous observations. We will slightly modify his statistic as follows; $J_{M}=\sum_{1 \times i<j=\mathrm{K}} U_{i j} / n_{i} n_{j}$, where $U_{i j}$ is a centralized Mann-Whitney statistic. Then for quantal responses, we obtain a test statistic for testing $\mathrm{H}_{0}$ against $\mathrm{K}_{0}$ as $\mathrm{J}_{M}-\sum_{\mathrm{k}=1}^{\mathrm{K}}(2 \mathrm{k}-1-\mathrm{K}) \mathrm{R}_{\mathrm{k}}$. Thus the statistic for testing $\mathrm{H}_{0}$ against $\mathrm{K}_{0}$ is a test statistic for testing $\delta=0$ against $\delta>0$, where

$$
\delta=\sum_{k=1}^{K}(2 k-1-K) p_{k} .
$$

The test statistic is identical to the Cochran-Armitage test , which is discussed below, with equi-spaced score $\theta\left(D_{\mathbf{k}}\right)=2 \mathbf{k}-1-\mathrm{K}, \mathbf{k}=\mathbf{1}, \cdots, \mathrm{K}$.

\subsection{Max Z test}

Kuriki, Hirotsu and Hayter (1989) proposed a test of a hypothesis of normal means $\mu_{1}, \cdots, \mu_{\mathrm{K}}$ that for some $\mathrm{k}, \mu_{1}=\cdots=\mu_{\mathrm{k}}<\mu_{\mathrm{k}+1}=\cdots=\mu_{\mathrm{k}}$. Hirotsu, Kuriki and Hayter (1992) proposed applying the same idea to the rank order test. The test is referred to as the max t-test, because the test statistic is based on the maximum of K-1 t-statistics. The same test statistic is considered by Ruberg (1989) using step contrasts. Their idea is immediately applied to our situation for testing $\mathrm{H}_{0}$ against $\mathrm{K}_{1}$ by considering a situation where the common variance is known. The resultant test statistic is $Z_{\max }=\operatorname{Max}\left(Z_{1}, \cdots, Z_{k-1}\right)$, where 


$$
Z_{k}=\frac{\left(R_{[k+1, K]}-R_{[1, k]}\right)}{\sqrt{R(1-R)\left(\frac{1}{n_{[k+1, K]}}+\frac{1}{n_{[1, k]}}\right)}}, \quad k=1, \cdots, K-1
$$

Critical points of $Z_{\max }$ for the cases $n_{1}=\cdots=n_{K}$ are obtained assuming asymptotic normality of $R_{1}=\cdots=R_{k}$ and are given by Kuriki, Hirotsu and Hayter (1989). When the sample sizes are not equal, exact asymptotic distribution should be calculated based on the formula given by Kuriki, Hirotsu and Hayter (1989) and Hirotsu, Kuriki and Hayter (1992). Ruberg (1989) gives conservative critical points. Let $Z_{\max }(\alpha)$ be upper $\alpha$ point of $Z_{\max }$ statistic calculated from Kuriki, Hirotsu and Hayter (1989) and Hirotsu, Kuriki and Hayter (1992). Then the large sample approximate test is defined as "reject $\mathrm{H}_{0}$ when $\mathrm{Z}_{\max } \geq \mathrm{Z}_{\max }(\alpha)$.

\subsection{Williams type test}

Williams (1971) proposed a stepwise test for determining the lowest dose level at which there is evidence for a difference from the control. A modification of his test for treating quantal response is the test of $\mathrm{H}_{0}$ against $\mathrm{K}_{2}$ and is defined by the statistic $\overline{\mathrm{Z}}$, where

$$
\overline{\mathrm{Z}}=\frac{\left(\mathrm{R}_{\mathrm{K}}-\mathrm{R}_{1}\right)}{\sqrt{\mathrm{R}(1-\mathrm{R})\left(\frac{1}{\mathrm{n}_{1}}+\frac{1}{\mathrm{n}_{\mathrm{K}}}\right)}}
$$

Then the large sample approximate test is defined by "reject $\mathrm{H}_{0}$ when $\bar{Z} \geq \bar{Z}(\alpha)$ ", where $\bar{Z}(\alpha)$ is the upper $\alpha$ point of Williams test statistic with infinite degrees of freedom, which is given in Williams (1971, 1972).

\subsection{A modified Williams type test}

A modification of Williams test was suggested by Williams (1971) and was examined by Marcus (1976). The test statistic can be modified for normal means with known variance. Thus we consider the following test statistic:

$$
\overline{\mathrm{Z}}_{\mathrm{M}}=\frac{\left(\mathrm{R}_{\mathrm{K}}^{\cdot}-\mathrm{R}_{1}^{*}\right)}{\sqrt{\mathrm{R}(1-\mathrm{R})\left(\frac{1}{\mathrm{n}_{1}}+\frac{1}{\mathrm{n}_{\mathrm{K}}}\right)}}
$$

Then the large sample approximate test is defined by "reject $H_{0}$ when $\bar{Z}_{M} \geq \bar{Z}_{M}(\alpha)$ ", where $\bar{Z}_{M}(\alpha)$ is the upper $\alpha$ point of modified Williams test statistic with infinite degrees of freedom, which is given in the table in Marcus (1976) for the case that all sample sizes are equal.

\subsection{Cochran-Armitage test}

Armitage (1955) and Cochran (1955) proposed a test of $\mathrm{H}_{0}$ against $\mathrm{K}_{3}$ for binomial populations. Let $\mathbf{x}=\left(\mathbf{x}_{1}, \cdots, \mathbf{x}_{\mathbf{k}}\right)$ be a given constant vector associated with dose levels like $\mathbf{x}_{\mathbf{k}}=\theta\left(\mathrm{D}_{\mathbf{k}}\right), \mathbf{k}=\mathbf{1}, \cdots, \mathbf{K}$, where $\theta\left({ }^{*}\right)$ is a monotone increasing function and satisfy that $x_{1} \leq \cdots \leq x_{K}$ and $x_{1}<x_{K}$. We refer to $x$ as a score vector for the Cochran-Armitage test. Then the test statistic is given by 


$$
Z=\frac{\sum_{k=1}^{K} r_{k} x_{k}-r \bar{x}}{\sqrt{\frac{r(n-r)}{n^{2}} \sum_{k=1}^{K} n_{k}\left(x_{k}-\bar{x}\right)^{2}}}
$$

where $\bar{x}=\sum_{k=1}^{K} n_{k} x_{k} / n$. This statistic has asymptotically standard normal distribution under $H_{0}$. Therefore $\mathrm{H}_{0}$ is rejected when $\mathrm{Z}$ is equal to or greater than $\mathrm{Z}(\alpha)$.

Cox (1958) shows that an equivalent test statistic is derived from a conditional argument for testing $\beta=0$ against $\beta>0$ when $\mathrm{p}_{\mathrm{k}}$ is given by a logistic model $\mathrm{p}_{\mathrm{k}}=1 /[1+\exp (-\alpha-\beta \theta$ $\left.\left.\left(D_{k}\right)\right)\right], k=1, \cdots, K$. Taron and Gart (1980) show that the test is a $C(\alpha)$ test for the model $p_{k}=H(\alpha+\beta \theta$ $\left.\left(D_{k}\right)\right), k=1, \cdots, K$, where $H$ is any monotone, and twice differentiable function.

\subsection{Dunnett type multiple comparison test}

A natural test of $\mathrm{H}_{0}$ against $\mathrm{K}_{4}$ is a Dunnett type multiple comparison. The test statistic is

$$
\mathrm{Z}_{\mathrm{D}}=\operatorname{Max}\left\{\mathrm{Z}_{2}, \cdots, \mathrm{Z}_{\mathrm{K}}\right\}
$$

where

$$
\mathrm{Z}_{\mathrm{k}}=\frac{\left(\mathrm{R}_{\mathrm{k}}-\mathrm{R}_{1}\right)}{\sqrt{\mathrm{R}(1-\mathrm{R})\left(\frac{1}{\mathrm{n}_{1}}+\frac{1}{\mathrm{n}_{\mathrm{k}}}\right)}}, \quad \mathrm{k}=2, \cdots, \mathrm{K}
$$

The test is defined as "reject $H_{0}$ when $Z_{D} \geq Z_{D}(\alpha ; K)$ ", where $Z_{D}(\alpha ; K)$ is the upper $\alpha$ point of Dunnett test with infinite degrees of freedom. The critical point is given at the line for infinite degrees of freedom given in the tables of Dunnett (1955), and Dunnett(1964). When the heterogeneity of sample sizes is large, Bonferroni type test can be used: "If $\mathrm{Z}_{0} \geq \mathrm{Z}\left(\frac{\alpha}{\mathrm{K}-1}\right)$ then reject $\mathrm{H}_{0}$ ", where $\mathrm{z}\left(\frac{\alpha}{\mathrm{K}-1}\right)$ is the upper $\frac{\alpha}{\mathrm{K}-1}$ point of the standard normal distribution. Although the hypothesis $\mathrm{K}_{4}$ does not imply monotonicity of the dose-response curve, the Dunnett test is often used for testing dose response problems. Therefore, it is valuable to examine this test. Of course, it is possible to detect departure from the null hypothesis $\mathrm{H}_{0}$ by using the Dunnett test when there really exists an appreciable dose-response relationship, and in some situations the Dunnett test may be powerful. However, caution is necessary in interpretation of the test results.

\subsection{Note on conditional test}

So far we defined an approximate unconditional test for every test discussed above. When sample sizes are small it is possible to adopt an exact test which is conditional on the observed total number of responses. For any test statistic $T(\mathbf{r})$ the probability is

Therefore when

$$
\operatorname{Pr}\left[T(\mathbf{r}) \mid \mathbf{n}, \mathbf{r}, \mathrm{H}_{\mathbf{0}}\right]=\frac{\mathrm{r} !(\mathrm{n}-\mathrm{r}) ! \prod_{\mathrm{k}=1}^{\mathrm{K}} \mathrm{n}_{\mathrm{k}} !}{\mathrm{n} ! \prod_{\mathrm{k}=1}^{K}\left\{\mathrm{r}_{\mathrm{k}} !\left(\mathrm{n}_{\mathrm{k}}-\mathrm{r}_{\mathrm{k}}\right) !\right\}}
$$

$$
\operatorname{Pr}\left[\mathrm{T}(\mathbf{R}) \geq \mathrm{T}(\mathbf{r}) \mid \mathbf{n}, \mathbf{r}, \mathrm{H}_{\mathbf{0}}\right]=\sum_{\mathbf{r} \in \mathbf{R}_{\mathbf{r}(r)}} \operatorname{Pr}\left[\mathrm{T}(\mathbf{r}) \mid \mathbf{n}, \mathbf{r}, \mathbf{H}_{\mathbf{0}}\right]
$$


is greater than $\alpha$ reject the null hypothesis. In what follows we discuss only the large sample approximate tests.

\section{Properties of test statistics and their exact unconditional distributions}

In this section, we study some properties of test statistics and their exact unconditional distributions. The observed numbers of responses $\mathrm{r}_{1, \ldots, \mathrm{rK}}$ jointly have product binomial distribution

$$
P(\mathbf{r} \mid \mathbf{n}, \mathbf{p})=\prod_{\mathrm{k}=1}^{\mathrm{K}}{ }_{\mathrm{n}_{\mathrm{k}}} \mathrm{C}_{\mathrm{r}_{\mathrm{k}}} \mathrm{p}_{\mathrm{k}}\left(1-\mathrm{p}_{\mathrm{k}}\right)^{\mathrm{n}_{\mathrm{k}}-\mathrm{r}_{\mathrm{k}}}
$$

Thus for the given $\mathbf{n}$ and $\mathbf{p}$ we can exactly calculate the distribution of any statistic $\mathbf{T}(\mathbf{R})$ defined in section 3 by the formula

$$
\begin{aligned}
\mathrm{F}_{\mathrm{T}}(\mathrm{t} \mid \mathbf{n}, \mathbf{p}) & =\operatorname{Pr}(\mathrm{T}(\mathrm{R}) \leq \mathrm{t}) \\
& =\sum_{\mathrm{r} \otimes_{\mathrm{T}(1)}} \mathrm{P}(\mathbf{r} \mid \mathbf{n}, \mathbf{p})
\end{aligned},
$$

where $R_{T(t)}$ is a set of $\mathbf{r}$ which satisfies $T(\mathbf{r}) \leq t$. We refer to the distribution $F_{T}(t \mid n, p)$ as exact unconditional distribution of a statistic $T(R)$ for given $\mathbf{n}$ and $\mathbf{p}$.

Now we introduce some terminology and notation. For given $\mathbf{p}, \mathbf{n}$ and $\mathbf{r}$, we write $\mathbf{p}^{\mathrm{R}}=\left(\mathrm{p}_{\mathrm{k}}, \cdots, \mathrm{p}_{1}\right), \mathbf{n}^{\mathrm{R}}=\left(\mathrm{n}_{\mathrm{k}}, \cdots \mathrm{n}_{1}\right)$ and $\mathbf{r}^{\mathrm{R}}=\left(\mathrm{rk}_{\mathrm{k}}, \cdots, \mathrm{r}_{1}\right)$, respectively, and refer to them as reverse of $\mathbf{p}, \mathbf{n}$ and $\mathbf{r}$, respectively. For given $\quad a=\left(a_{1}, \cdots, a_{k}\right)$ where $0 \leqq a_{k} \leqq 1, k=1, \cdots, K$, there corresponds a K-tuple $\mathbf{b}=\left(b_{1}, \cdots, b_{k}\right)$ where $b_{k}=1-a_{k+1-k}, k=1, \cdots, K$. We refer to $b$ as the conjugate of $a$ and denote it by $a^{c}$. For the observed frequency vector $r$, we define the conjugate of $r$, denoted by $r^{c}$, as $r^{c}=n^{R}-r^{R}$. Let $\mathbf{x}=\left(\mathrm{x}_{1}, \cdots, \mathrm{x}_{\mathrm{K}}\right)$ be any score vector for the Cochran-Armitage test, and let $\mathbf{x}^{\mathrm{C}}(\xi)=\left(\xi-\mathrm{x}_{\mathrm{K}}, \cdots, \xi-\mathrm{x}_{1}\right)$, where $\xi$ is any given constant. Let $R$ and $R^{\star}$ be the vectors of raw and max-min estimates of $p$ as defined in section 2 . We easily have the following propositions.

Proposition 1: The test statistics of the chi-square test, Jonckheere type test and the max- $\mathrm{Z}$ test have the property that $T(R)=T\left(R^{c}\right)$.

Proposition 2: Let $T(R \mid \mathbf{x})$ and $T\left(R^{c}(\xi) \mid \mathbf{x}^{c}\right)$ be Cochran-Armitage test statistics whose scores are $\mathbf{x}$ and $\mathbf{x}^{c}$ $(\xi)$, respectively, then $T(R \mid \mathbf{x})=T\left(R^{c}(\xi) \mid x^{c}\right)$ holds. For an equi-spaced score $\mathbf{x}, T(R \mid \mathbf{x})=T\left(R^{c} \mid \mathbf{x}^{c}\right)$ holds.

Proposition 3: The chi-bar square statistic and modified Williams statistic have the property that $T\left(R^{*}\right)=T\left(R^{*}\right)$.

Proofs of these propositions are trivial, so omitted. Note that the Williams type test statistic does not satisfy these properties. For example, suppose $\mathbf{n}=(10,10,10,10)$ and $\mathbf{r}=(3,1,7,9)$, then $\mathrm{R}=(0.3,0.1,0.7,0.9)$ and $R^{*}=(0.2,0.2,0.7,0.9)$, thus $\bar{z}=(0.9-0.1) /\left\{0.5^{2}(1 / 10+1 / 10)\right\}^{1 / 2}$ for $R$. Whereas $R^{C}=(0.1,0.3,0.9,0.7)$ and $\mathrm{R}^{* \mathrm{C}}=(0.1,0.3,0.8,0.8)$ which give $\overline{\mathrm{z}}=(0.8-0.1) /\left\{0.5^{2}(1 / 10+1 / 10)\right\}^{1 / 2}$. Contrary to the Williams type test statistic, the modified Williams type test statistic gives the same $\bar{z}_{\mu}$ values.

Suppose that $K^{*}$ is one of the hypotheses $K_{0}, K_{1}, K_{2}, K_{3}$ and $K_{4}$, and $p$ satisfies $K_{*}$, then the conjugate of $p$ also satisfies $K *$. Let $(n, p)$ stand for an experiment where $n_{k}$ subjects are administered dose $\mathrm{D}_{\mathbf{k}}$ with response probability $\mathbf{p}_{\mathbf{k}}, \mathbf{k}=1, \cdots, K$. What we want to show is a sufficient condition that two experiments $(\mathbf{n}, \mathbf{p})$ and $(\mathbf{m}, \mathbf{q})$ give the same distribution of a given test statistic for testing $\mathrm{H}_{0}$ against $\mathrm{K}^{*}$. The main result is stated in the Theorem 1 and Theorem 2 . For showing Theorem 1, first we show Proposition 4.

Proposition 4: The following equality holds:

$$
\mathrm{P}(\mathbf{r} \mid \mathbf{n}, \mathbf{p})=\mathrm{P}\left(\mathbf{r}^{\mathrm{c}} \mid \mathbf{n}^{\mathrm{R}}, \mathbf{p}^{\mathrm{c}}\right) .
$$

Proof Let $q=\left(q_{1}, \ldots, q K\right)=p^{c}, s=\left(s_{1}, \ldots, S K\right)=r^{c}$, then we have 


$$
\begin{aligned}
P\left(\mathbf{r}^{c} \mid \mathbf{n}^{\mathrm{R}}, \mathbf{p}^{\mathrm{c}}\right) & =\prod_{\mathrm{k}=1}^{\mathrm{K}}{ }_{n_{K+1-k}} C_{s_{k}} q_{k}^{s_{k}}\left(1-q_{k}\right)^{n_{K+1-k}-s_{k}} \\
& =\prod_{k=1}^{K}{ }_{n_{K+1-k}} C_{n_{K+1-k}-r_{K}+1-k}\left(1-p_{K+1-k}\right)^{n_{K}+1-k-r_{K}+1-k} p_{K+1-k}^{r_{K}+1-k} \\
& =\prod_{h=1}^{K}{ }_{n_{h}} C_{T_{h}} p^{r_{h}}\left(1-p_{h}\right)^{n_{h}-r_{h}} \\
& =P(\mathbf{r} \mid \mathbf{n}, p)
\end{aligned}
$$

Thus the proposition was shown.

Theorem 1: Let $T=T(R)$ be a statistic of $R$ satisfying $T(R)=T\left(R^{c}\right)$. Then the exact unconditional distribution of $T$ under the experiment $(\mathbf{n}, \mathbf{p})$ is identical to that under the experiment $\left(\mathbf{n}^{\mathrm{R}}, \mathbf{p}^{\mathrm{c}}\right)$, that is, $F_{T}(t \mid n, p)=F_{T}\left(t \mid n^{R}, p^{c}\right)$.

Proof: Let $\mathbf{r}$ be any sample from $(\mathbf{n}, \mathbf{p})$. Then the sample $\mathbf{r}^{c}$ from the experiment $\left(\mathbf{n}^{\mathrm{R}}, \mathbf{p}^{\mathrm{c}}\right)$ has the same probability as $\mathbf{r}$. Furthermore $R$ and $R^{c}$ are derived from $\mathbf{r}$ and $r^{c}$, respectively and give the same statistic $T(R)=T\left(R^{c}\right)$.

Let $R_{T(t)}$ be the set of $r$ giving $T(R) \leqq t$. Then

$$
\begin{aligned}
& P(T(R) \leqq \mid(\mathbf{n}, \mathbf{p}))=\sum_{r \in \mathbb{R}_{T(t)}} P(\mathbf{r} \mid \mathbf{n}, \mathbf{p}) \\
& =\sum_{r \in R_{T(t)}} \mathrm{P}\left(\mathbf{r}^{c} \mid \mathbf{n}^{\mathrm{R}}, \mathbf{p}^{c}\right) \\
& =P\left(T\left(R^{c}\right) \leqq t \mid\left(\mathbf{n}^{R}, p^{c}\right)\right) \text {. }
\end{aligned}
$$

Thus the assertion is proved.

Similar statement holds for a statistic defined by $\mathbf{R}^{*}$.

Proposition 5: Let $s=\left(s_{1}, \cdots, s_{K}\right)$ be the conjugate of $r$. Then the max-min estimate of $q$ under $K_{0}$ based on $\mathbf{s}$ is given by $\mathbf{R}^{*}$.

Proof: Denote $Q_{k}^{*}$ as the max-min estimate of $q_{k}$ based on $r^{c}$. Then we have

$$
\begin{aligned}
& Q_{k}^{*}=\operatorname{Max}_{1 \leq u \leq k} \operatorname{Min}\left(1-\frac{r_{[K+1-v, K+1-u]}}{n_{[K+1-v, K+1-u]}}\right) \\
& =\operatorname{Max}_{1 \leq u \leq k}\left(1-\operatorname{Max}_{\mathbf{k} \leq v \leq K} \frac{r_{[K+1-v, K+1-u]}}{n_{[K+1-v, K+1-u]}}\right) \\
& =1-\operatorname{Min}_{1 \leq u \leq k} \operatorname{Max}_{k \leq v \leq K} \frac{r_{[K+1-v, K+1-u]}}{n_{[K+1-v, K+1-u]}} \\
& =1-\operatorname{Min}_{K+1-k \leq u \leq K} \operatorname{Max}_{1 \leq v \leq K+1-k} \frac{r_{[u, v]}}{n_{[u, v]}} \\
& =1-\operatorname{Max}_{1 \leq u \leq K+1-k} \operatorname{Min}_{K+1-k \leq v \leq K} \frac{r_{[u, v]}}{n_{[u, v]}} \\
& =1-\mathrm{R}
\end{aligned}
$$

Thus the proposition is shown.

Theorem 2: Let $T=T\left(R^{*}\right)$ be a statistic of $R^{*}$ satisfying $T\left(R^{*}\right)=T\left(R^{*}\right)$. Then the exact unconditional distribution function of $T$ under the experiment $(\mathbf{n}, \mathbf{p})$ is identical to that under the experiment $\left(\mathbf{n}^{\mathrm{R}}, \mathbf{p}^{\mathrm{c}}\right)$, 
that is, $F_{T}(t \mid \mathbf{n}, \mathbf{p})=F_{T}\left(t \mid \mathbf{n}^{R}, p^{c}\right)$ holds.

Proof Let $\mathbf{r}$ be any sample from $(\mathbf{n}, \mathbf{p})$. Then $\mathbf{r}^{c}$ is the sample from $\left(\mathbf{n}^{\mathrm{R}}, \mathbf{p}^{c}\right)$ which gives the same probability element as that of $r$. Since $R^{*}$ and $R^{*}$ give the same value of statistic $T$ for the set of samples $\mathbf{R}^{*}(t)=\left\{r ; T\left(R^{*}\right) \leqq t\right\}$, next equality holds;

$$
\begin{aligned}
\mathrm{P}\left(\mathrm{T}\left(\mathrm{R}^{*}\right) \leqq \mathrm{t} \mid(\mathbf{n}, \mathbf{p})\right) & =\sum_{\mathbf{r} \in \mathbb{R}_{T_{(t)}}} \mathrm{P}(\mathbf{r} \mid(\mathbf{n}, \mathbf{p})) \\
& =\sum_{\left.r \in \mathbb{R}_{(}^{*}\right)} \mathrm{P}\left(\mathbf{r}^{\mathrm{c}} \mid\left(\mathbf{n}^{\mathrm{R}}, \mathbf{p}^{\mathrm{c}}\right)\right) \\
& =\mathrm{P}\left(\mathrm{T}\left(\mathbf{R}^{*} \mathrm{c}\right) \leqq \mathrm{t} \mid\left(\mathbf{n}^{\mathrm{R}}, \mathbf{p}^{\mathrm{c}}\right)\right) .
\end{aligned}
$$

Thus the assertion of the theorem 2 was proved.

From the above theorems and proposition 1,2 and 3, it follows that each of the test statistics of the chi-square test, chi-bar square test, Jonckheere type test, Cochran-Armitage test with equi-spaced score, Max-Z test and modified Williams type test have the same distribution for $(\mathbf{n}, \mathbf{p})$ and $\left(\mathbf{n}^{\mathrm{R}}, \mathbf{p}^{\mathrm{c}}\right)$. Thus the chi-bar square test, for example, has the same power for the alternative $p=(0.2,0.3,0.7)$ with samples $\mathrm{n}=(15,17,13)$ as for the alternative $\mathrm{p}=(0.3,0.7,0.8)$ with samples $\mathrm{n}=(13,17,15)$.

\section{Example}

Let us apply these tests to actual dose-response studies. Table 1 shows rates of patients who showed sufficient reduction of heart rates when administered $2.5 \mathrm{mg}, 5.0 \mathrm{mg}$ or $10 \mathrm{mg}$ of diltiazem under operation. Maximum likelihood estimates of $R_{1}, R_{2}$ and $R_{4}$ under $K_{0}$ are $0.3200,5703$ and 0.5703 , respectively, and $R=0.4722$. We have

$$
\begin{aligned}
& \bar{\chi}^{2}=8.702>\bar{\chi}^{2}(0.01 ; 3)=6.822, \\
& \mathrm{Z}_{\max }=2.950>\mathrm{Z}_{\max }(0.01 ; 3)=2.588, \\
& \overline{\mathrm{Z}}=2.572>\overline{\mathrm{Z}}(0.01 ; 3)=2.366, \\
& \overline{\mathrm{Z}}_{\mathrm{M}}=2.572>\overline{\mathrm{Z}}_{\mathrm{M}}(0.01 ; 3)=2.400, \\
& \mathrm{Z}=2.938>\mathrm{Z}_{\mathrm{D}}(0.01 ; 3), \\
& \mathrm{Z}=2.146>\mathrm{Z}(0.025)=1.960 .
\end{aligned}
$$

Thus every test except for the Cochran-Armitage test indicates that the dose-response is significant at one percent level of significance of one-sided test. The Cochrane-Armitage test is significant at five percent level of significance. $\mathrm{Z}_{\mathrm{Max}}$ is given by $\mathrm{Z}_{1}$ and $\mathrm{Z}_{\mathrm{D}}$ is given by $\mathrm{Z}_{2}$. Every test indicates that $5.0 \mathrm{mg}$ is more effective than $2.5 \mathrm{mg}$. The chi-square test statistic for testing equality of response rates of $5.0 \mathrm{mg}$ and $10 \mathrm{mg}$ is 0.533 . This indicates that the results that $10 \mathrm{mg}$ gave lower response rate than $5 \mathrm{mg}$ is highly possible by chance. Thus it can be concluded that $5 \mathrm{mg}$ is more effective than $2.5 \mathrm{mg}$ and is equivalent to $10 \mathrm{mg}$, that is, $5 \mathrm{mg}$ is a plateau dose.

Table 1. Effect of diltiazem on heart rate reduction

\begin{tabular}{ccccc}
\hline Dose & \multicolumn{3}{c}{ number of subjects } & response rate \\
\cline { 2 - 4 } & effective & not effective & total & $(\%)$ \\
\hline $2.5 \mathrm{mg}$ & 16 & 34 & 34 & 32.0 \\
$5.0 \mathrm{mg}$ & 35 & 23 & 23 & 60.3 \\
$10.0 \mathrm{mg}$ & 30 & 26 & 26 & 53.6 \\
\hline
\end{tabular}

6. Type I error rates of the large sample approximate tests

We derived large sample approximate tests of $\mathrm{H}_{0}$ against ordered alternatives. However, due to 
their discrete nature of response variables, and since they are asymptotically valid, the sample sizes may affect their validity. That is, when the sample sizes are small or moderate, the actual type I error rates possibly differ from the nominal significance level. It is worthwhile to examine agreement of the actual type I error rates and the nominal significance levels. For this purpose we calculate type I error rates by exact enumeration of the unconditional probability function $\mathrm{F}_{\mathrm{r}}(\mathrm{t} \mid \mathbf{n}, \mathbf{p})$ at the nominal level 0.05 , and 0.01 . That is, the exact type I error rate is calculated by $1-\mathrm{F}_{\mathrm{T}}(\mathrm{t}(\alpha) \mid \mathbf{n}, \mathbf{p})$, where $t(\alpha)$ is the upper $\alpha$ point described in section 3. Since the number of dose levels in a dose-response study is mostly three or four, we show the

Table 2. Type I error rate of the unconditional tests for trend in case of 3 populations and equal sample sizes of $n_{0}$.

\begin{tabular}{|c|c|c|c|c|c|c|c|c|c|}
\hline $\mathrm{n}_{0}$ & $\mathrm{p}$ & $\alpha$ & $x^{2}$ & $\bar{x}^{2}$ & $\mathrm{Z}$ & $Z_{\max }$ & $\mathrm{Z}_{\mathrm{D}}$ & $\overline{\mathrm{Z}}$ & $\overline{\mathbf{Z}}_{\mathbf{M}}$ \\
\hline \multirow{8}{*}{20} & .20 & 5.0 & 4.98 & 5.04 & 4.91 & 4.80 & 5.10 & 5.09 & 5.19 \\
\hline & & 1.0 & $.85--$ & .98 & .91 & .89-- & .87-- & .95 & .87 \\
\hline & .30 & 5.0 & 5.22 & 5.09 & $5.43+$ & 4.99 & $5.39+$ & 5.10 & 5.07 \\
\hline & & 1.0 & .89 & 1.03 & 1.00 & $.90-$ & .99 & .99 & .96 \\
\hline & .40 & 5.0 & 5.00 & 5.18 & 4.58 & 4.98 & $5.47+$ & 4.77 & 4.98 \\
\hline & & 1.0 & .90 & 1.00 & .87-- & 1.04 & $1.06+$ & $.92-$ & .95 \\
\hline & .50 & 5.0 & 4.96 & 5.14 & 4.13-- & 5.01 & 4.39-- & 4.96 & 4.99 \\
\hline & & 1.0 & $.88--$ & .94 & .84-- & $1.08+$ & .83-- & .97 & .98 \\
\hline \multirow{8}{*}{30} & .20 & 5.0 & 4.90 & 5.00 & 5.18 & 4.96 & 5.23 & 4.99 & 5.09 \\
\hline & & 1.0 & $4.90-$ & .98 & .98 & .88-- & 1.02 & $92-$ & .98 \\
\hline & .30 & 5.0 & 5.17 & 4.97 & 5.22 & 4.95 & 5.23 & 4.91 & 4.86 \\
\hline & & 1.0 & .98 & .99 & 1.00 & $.94-$ & 1.02 & 1.00 & 1.02 \\
\hline & .40 & 5.0 & 5.06 & 5.12 & 4.51 - & 5.20 & 4.58 & 5.00 & $5.36+$ \\
\hline & & 1.0 & .96 & 1.01 & $1.17++$ & 1.01 & $1.13++$ & 1.00 & .94 \\
\hline & .50 & 5.0 & 4.80 & 4.98 & $4.63-$ & $5.57++$ & 4.71 & 5.19 & $5.72++$ \\
\hline & & 1.0 & .97 & .98 & $1.08+$ & $.91-$ & $1.28++$ & $.82-$ & $.88--$ \\
\hline \multirow{8}{*}{50} & .20 & 5.0 & 4.93 & 5.07 & 5.07 & 4.99 & 5.15 & 5.02 & 4.92 \\
\hline & & 1.0 & .96 & .95 & .99 & .99 & 1.01 & .95 & .98 \\
\hline & .30 & 5.0 & 5.01 & 5.10 & 5.12 & 5.11 & 5.16 & 5.06 & 4.94 \\
\hline & & 1.0 & .97 & .97 & 1.01 & .99 & 1.00 & .99 & 1.00 \\
\hline & .40 & 5.0 & 5.22 & 5.09 & 4.82 & 4.98 & 4.81 & 4.87 & 5.22 \\
\hline & & 1.0 & 1.01 & .99 & .95 & 1.03 & 1.01 & 1.02 & $1.10+$ \\
\hline & .50 & 5.0 & 4.80 & $5.41+$ & 4.44-- & 5.12 & 5.14 & 5.02 & $5.55++$ \\
\hline & & 1.0 & .96 & 1.02 & 1.05 & $.91-$ & $1.14++$ & $1.14++$ & $1.22++$ \\
\hline
\end{tabular}

$\mathrm{p}$ stands for the common response rate.

results of $\mathrm{K}=3$ and 4 in Table 2 and Table 3, respectively. We show only the cases where $\mathrm{K}$ samples have the same size, $n_{0}=20,30$ and 50 . The response probabilities of null hypotheses examined are $0.2,0.3,0.4$ and 0.5 . The results are the same for $0.6,0.7,0.8$ as for $0.4,0.3$ and 0.2 , respectively. This fact results from Theorem 1 and Theorem 2

Since the exact distribution of any statistic is discrete, it is usually impossible for any test to exactly attain the nominal level. So we accept the relative error less than five percent of the given nominal level. In Table 2 and 3, the super scripts + and ++ indicate that the actual level exceeds the nominal level by more than 5 percent and 10 percent of the nominal level, respectively. The symbols and -- indicate that the actual level is less than the nominal level more than 5 percent and 10 percent of the nominal level, respectively. The results of every test are summarized as follows.

(1) Chi-square test : The test is acceptable when no is equal to or greater than 20.

(2) Chi-bar square test : The type I error rate is more stable than the other tests studied. Even when no is equal to twenty, it keeps type I error rate at almost the nominal level. 
UESAKA

Table 3. Type I error rate of the unconditional tests for trend in case of 4 populations and equal sample sizes of $n_{0}$.

\begin{tabular}{|c|c|c|c|c|c|c|c|c|c|}
\hline no & $\bar{p}$ & $\alpha$ & $x^{2}$ & $\bar{x}^{2}$ & $\mathrm{Z}$ & $Z_{\max }$ & $\mathrm{ZD}_{\mathrm{D}}$ & $\overline{\mathbf{Z}}$ & $\overline{\bar{Z}}_{\mathrm{M}}$ \\
\hline \multirow{8}{*}{20} & .20 & 5.0 & 4.77 & 5.15 & 5.25 & 4.93 & $5.56++$ & 5.06 & 5.06 \\
\hline & & 1.0 & .87-- & .96 & $.93-$ & .92 & .99 & .87-- & .96 \\
\hline & .30 & 5.0 & 5.05 & 5.18 & 5.18 & 5.00 & $5.37+$ & 5.07 & 4.93 \\
\hline & & 1.0 & .91 & 1.00 & .97 & .96 & 1.03 & .96 & $.94-$ \\
\hline & .40 & 5.0 & 4.99 & 4.96 & 5.07 & 5.20 & $4.72-$ & 4.85 & 5.09 \\
\hline & & 1.0 & .91- & .97 & 1.00 & 1.04 & .86-- & .90 & .94 \\
\hline & .50 & 5.0 & 4.87 & 4.96 & 4.99 & 5.25 & 4.92 & 5.13 & $5.25+$ \\
\hline & & 1.0 & $.94-$ & $.95-$ & .96 & $1.12++$ & .89-- & .96 & 1.00 \\
\hline \multirow{8}{*}{30} & .20 & 5.0 & 4.93 & 4.92 & 5.04 & 5.20 & $5.46+$ & 4.91 & 4.97 \\
\hline & & 1.0 & .89-- & .97 & .96 & .88-- & 1.02 & .97 & .93 \\
\hline & .30 & 5.0 & 5.05 & 4.99 & 5.07 & 5.00 & 5.14 & 4.82 & 4.95 \\
\hline & & 1.0 & .94- & .98 & .99 & .97 & $1.06+$ & .97 & .98 \\
\hline & .40 & 5.0 & 4.96 & 5.02 & 5.12 & 4.89 & $6.06++$ & 5.13 & $5.30+$ \\
\hline & & 1.0 & .97 & .99 & .99 & .97 & .89-- & .97 & .89- \\
\hline & .50 & 5.0 & 5.06 & 4.96 & 4.88 & 4.84 & $6.50++$ & $5.37+$ & 4.41 \\
\hline & & 1.0 & .97 & .96 & .99 & .98 & .86-- & .82-- & .92 \\
\hline \multirow{8}{*}{50} & .20 & 5.0 & 4.90 & 5.01 & 5.16 & 4.86 & $5.39+$ & 4.96 & 4.95 \\
\hline & & 1.0 & .95 & .97 & .99 & .99 & 1.02 & .94 & .95 \\
\hline & .30 & 5.0 & 4.96 & 5.08 & 5.05 & 5.12 & 5.19 & 5.01 & 4.91 \\
\hline & & 1.0 & .97 & .99 & 1.00 & 1.00 & 1.02 & .99 & .95 \\
\hline & .40 & 5.0 & 5.02 & 5.07 & 5.07 & 5.03 & 4.87 & 5.00 & 5.13 \\
\hline & & 1.0 & .96 & .99 & 1.00 & 1.00 & $.94-$ & 1.04 & 1.02 \\
\hline & .50 & 5.0 & 5.12 & 4.98 & 5.13 & 4.88 & 4.53- & 5.22 & $4.42-$ \\
\hline & & 1.0 & .97 & 1.04 & 1.04 & $1.09+$ & .92 & $1.17++$ & $.83--$ \\
\hline
\end{tabular}

$\mathrm{p}$ stands for the common response rate.

(3) Cochran-Armitage test : When $\mathrm{K}$ is four, it well keeps the nominal levels even when $\mathrm{n}_{0}$ is equal to twenty. However, when $\mathrm{K}$ is three, it is not so stable as chi-bar square test and is slightly conservative. This is because the test statistic for 3 samples case is actually a test of difference of proportions between the first and the third samples due to the equi-spaced scores. Thus except for the error variance there is no contribution of the second sample, which results in slow convergence to the asymptotic distribution. In case of four samples, all samples contribute to the test statistic, thus convergence is faster compared to the three sample case.

(4) Max-Z test : The results show that the Max-Z test is not so stable as chi-bar square test but more stable than Cochran-Armitage test.

(5) Dunnett type test : The type I error rate seems fairly unstable even the size of each sample is fifty. One reason for the slow convergence to the asymptotic distribution may be that the test is essentially a two-sample test except for the estimates of variance. Therefore there are no contributions of other samples than the relevant pair of samples. This fact results in the greater discreteness of probability distribution.

(6) Williams type test : When no is equal to or greater than thirty the actual type I error rate is close to the nominal level. The second sample contributes to the test statistic to some extent, but the slow approximation to the asymptotic distribution is due to the isolate use of the first sample.

(7) Modified Williams type test : The type I error rate of the modified Williams test is not so stable as that of the chi-bar square test. It is difficult to know why the type I error rate is so high for some cases even when sample sizes are 50.

It is often said that for small samples, an exact test conditional on the given marginal totals should be used in order to avoid dependence of the type I error rate on the null response probabilities. However, since the actual type I error rate is between $95 \%$ and $105 \%$ of the nominal level for most response probabilities, the large sample approximate tests seem applicable for samples of which sizes are as large 
as twenty.

\section{Power comparisons}

In this section, we examine powers of the tests considered in section 3. Since the number of doses tested in clinical dose-response trials are usually three or four, we examined various non-decreasing dose-response patterns with dose levels of three or four. The power is given by $\mathrm{P}(\mathrm{T}(\mathbf{R}) \geq \mathrm{t}(\alpha))$ for given $\mathbf{p}$ and $\mathbf{n}$, which is obtained by exactly enumerating the product binomial probabilities as is in section 6 . Among huge number of cases examined, only typical results are given in Fig.1, Fig.2 and Table 4. The significance level is five percent. In Table 4, to identify powerful tests, the highest value among the six tests is underlined and values which do not differ from the highest values by more than one percent are highlighted by asterisks.

It is possible to identify which test is most powerful for some of the alternatives. For example for $\mathrm{K}_{3}$, the Cochran-Armitage test should be most powerful. For $\mathrm{K}_{1}$, the max-Z test should be most powerful. For $\mathrm{K}_{4}$ where $\mathrm{p}_{1}<\mathrm{p}_{2}=\cdots=\mathrm{p}_{\mathrm{K}}$, the Dunnett type test seems to be very powerful, because all K-1 samples equally contribute to rejecting the null hypothesis. However, there are many situations where pattern of $p$ is between these typical patterns. For such a pattern it is very difficult to predict which test is the most powerful. For quantitatively describing how the tests perform for a typical pattern of $p$, numerical comparisons by exact calculation of powers is inevitable.

First, let us summarize findings about cases of three dose-levels. Fig. 1 and Fig.2 show power curves as functions of $p_{2}$ when $p_{2}$ changes from $p_{1}$ to $p_{3}$. Figure 1 and Figure 2 show the case that $\mathrm{p}_{1}=0.20, \mathrm{p}_{3}=0.40, \mathrm{n}=(60,60,60)$ and the case $\mathrm{p}_{1}=0.20, \mathrm{p}_{3}=0.50, \mathrm{n}=(30,30,30)$, respectively.

(1) For the alternative $K_{3}$, the Cochran-Armitage test is expected to give the highest power. However, the modified Williams type test and Williams type test often give a power as high as that of the Cochran-Armitage test. These three tests seem to be equally powerful against $\mathrm{K}_{3}$.

(2) For the case of $K_{1}$, that is, $p_{1}=p_{2}<p_{3}$ or $p_{1}<p_{2}=p_{3}$, the chi-bar square test is always better than the Cochran-Armitage test. The Max- $\mathrm{Z}$ test and the modified Williams type test seem as powerful as the chi-bar square test as expected. It should be noted that for any $p$ satisfying $K_{1}$, $p^{c}$ also satisfies $K_{1}$, and each of the Max-Z test, modified Williams type test and chi-bar square test individually gives the same power for $\mathbf{p}$ and $\mathbf{p}^{\mathrm{c}}$.

(3) The Williams type test and Dunnett type test are as powerful as chi-bar square test when $p_{1}<p_{2}=p_{3}$, but they are considerably inferior to others when $p_{1}=p_{2}<p_{3}$.

(4) The Williams type test is the most powerful when $p_{2}$ is between $\left(p_{1}+p_{3}\right) / 2$ and $p_{3}$. Almost the same feature is observed for several charts obtained by changing $p_{2}$ from $p_{1}$ to $p_{3}$. It can be said that when $p_{2}$ is greater than $\left(\mathrm{p}_{1}+\mathrm{p}_{3}\right) / 2$ William test is recommended.

(5) The chi-bar square, modified Williams type and Max-Z tests are similar to each other. Chi-bar square test and modified Williams type test keep relatively higher power for any particular hypothesis.

Next we consider the case of four doses. The results are given in Table 4. The properties of the tests are more remarkable.

(1) For $\mathrm{K}_{3}$ the Cochran-Armitage test is most powerful as expected. The Williams type test and Modified-Williams type test are ranked second. Chi-bar square is slightly less powerful than these tests.

(2) When $p_{1}=p_{2}=p_{3}<p_{4}$, chi-bar square test, Max- $Z$ test and modified Williams type test give almost the same power, but other tests are considerably inferior to the above three tests.

(3) When $p_{1}<p_{2}=p_{3}=p_{4}$ the Dunnett type test and the Williams type test are most powerful, and chi-bar square test, Max-Z test and the modified Williams type test are somewhat inferior to the above two tests. The Cochran-Armitage test is considerably inferior to others.

(4) When $\mathrm{p}_{1}<\mathrm{p}_{2} \leqq \mathrm{p}_{3} \leqq \mathrm{p}_{4}$, the Williams type test is most powerful.

(5) When $p_{1}=p_{2}<p_{3}=p_{4}$, the Max- $Z$ test is expected to be most powerful. The chi-bar square test and the Cochran-Armitage test give as high power as the Max- $Z$ test. The modified Williams type test is somewhat inferior to the above three tests.

(6) When $\mathrm{p}_{1}=\mathrm{p}_{2}<\mathrm{p}_{3}<\mathrm{p}_{4}$, the chi-bar square and Max-Z tests seem generally powerful, modified Williams 


\section{UESAKA}

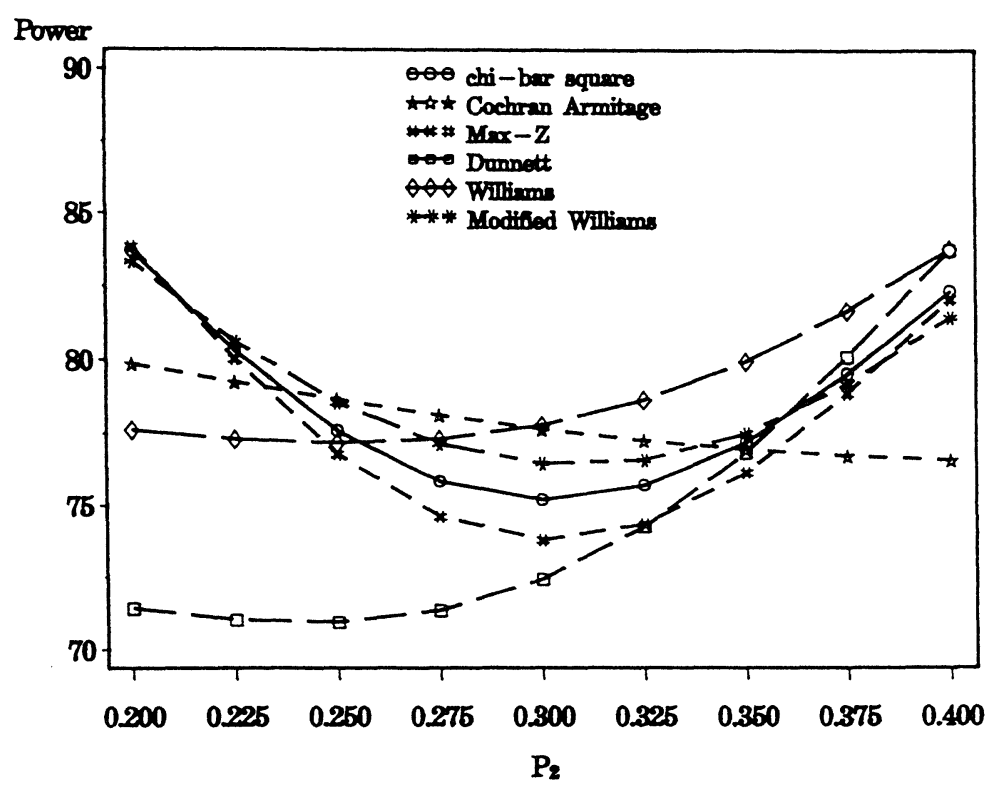

Figure 1 Power curves of several tests with $\mathrm{P}_{2}$ changing from $\mathrm{P}_{1}$ to $\mathrm{P}_{2}$ $\left(n_{1}=n_{2}=n_{8}=60\right)$

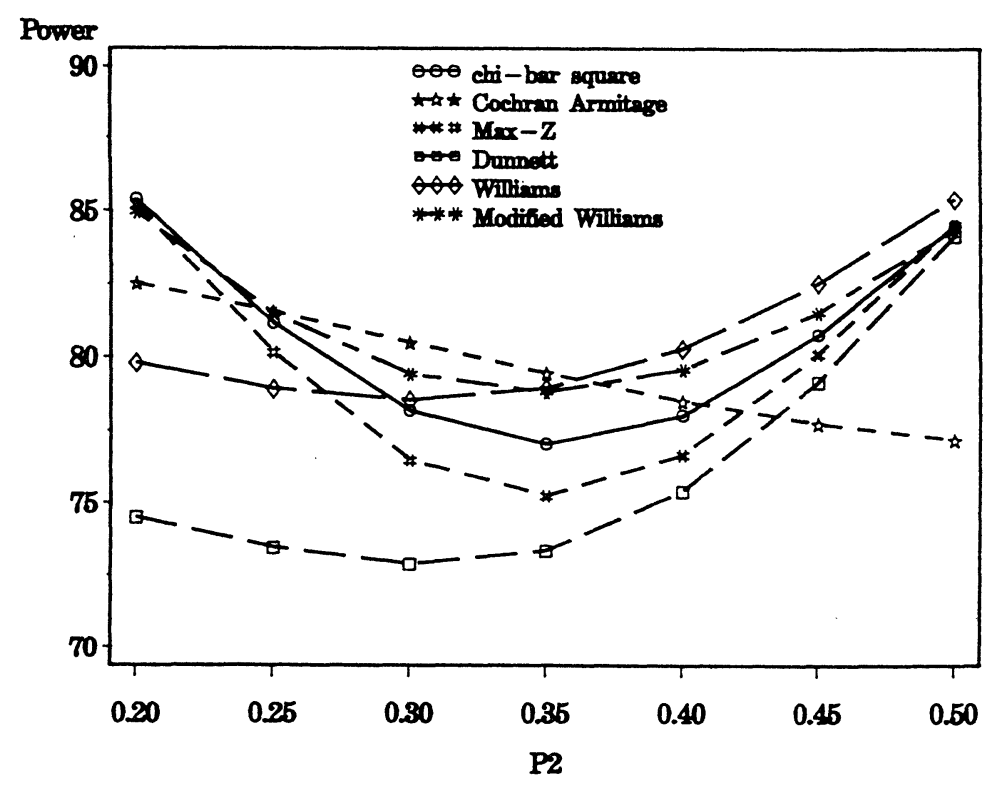

Figure 2. Power curves of several tests with $\mathrm{P}_{2}$ changing from $\mathrm{P}_{1}$ to $\mathrm{P}_{2}$

$$
\left(n_{1}=n_{2}=n_{3}=30\right)
$$


type test is also powerful. The power of the Cochran-Armitage test changes depending on departure from linearity.

It may be concluded that 1 ) for $\mathrm{K}_{1}$, the chi-bar square test and Max- $Z$ test are generally suitable, and 2) for $\mathrm{K}_{2}$, the chi-bar square, the Cochran-Armitage, the Max- $\mathrm{Z}$ and the modified Williams type tests are comparable.

\section{Determination of test procedure and sample sizes for a dose-response study \\ 8.1 General procedure}

Approximate formulae for calculating sample size are available for the chi-square test and the Cochran-Armitage test. But for other tests no formulae are available. Thus

we calculate exact unconditional power of the given test for several n's by the method described in the previous sections, and search for the appropriate $n$. When $\mathbf{n}=\mathrm{n}_{0}\left(\mathrm{n}_{1}, \ldots, \mathrm{nK}\right)$, we can get the required sample size in the following manner. First, select several $\mathbf{n}_{0}$, say $n_{0}, i=1, \ldots, I$, and compute powers $P\left(n_{0 i}\right)$, $\mathrm{i}=1, \ldots, \mathrm{I}$, thus we get pairs $\left(\mathrm{n}_{0}, \mathrm{P}\left(\mathrm{n}_{\mathrm{i}}\right)\right), \mathrm{i}=1, \ldots, \mathrm{I}$. Next, fit a polynomial to the power plot $\left(\mathrm{n}_{0 \mathrm{i}}, \mathrm{P}\left(\mathrm{n}_{0 \mathrm{i}}\right)\right), \mathrm{i}=1, \ldots, \mathrm{I}$ and then get the solution to the equation that the polynomial value is equal to the required power. The first approximate $n_{0}$, say $\mathrm{n}_{A}$ is obtained as the minimum integer which is not less than the solution to the above equation. The sample size vector $n_{A}\left(n_{1}, \ldots, n_{K}\right)$ is an estimate of the sample sizes. Unfortunately the power function is not necessarily monotonic with respect to $\mathrm{n}_{0}$, so it is better to calculate the powers for consecutive $n_{0}$ around $n_{A}$ to get a more precise value.

Table 4. Powers of tests in case of four dose-groups at $5 \%$ level of significance and equal sample sizes.

\begin{tabular}{|c|c|c|c|c|c|c|c|c|c|c|c|}
\hline $\mathrm{n}_{0}$ & $\mathrm{p}_{1}$ & $\mathrm{p}_{2}$ & $\mathrm{p}_{3}$ & $\mathrm{p}_{4}$ & $x^{2}$ & $\bar{x}^{2}$ & $\mathrm{Z}$ & $\mathrm{Z}_{\max }$ & $\mathrm{Z}_{\mathrm{D}}$ & $\mathrm{Z}$ & $\overline{\mathrm{Z}}_{\mathrm{M}}$ \\
\hline \multirow{8}{*}{20} & 0.1 & 0.2 & 0.3 & 0.4 & 47.38 & 73.05 & 77.43 & 69.58 & 66.75 & 75.34 & 73.79 \\
\hline & 0.2 & 0.3 & 0.4 & 0.5 & 39.25 & 64.27 & 69.21 & 62.18 & 54.39 & 64.32 & 64.88 \\
\hline & 0.3 & 0.4 & 0.5 & 0.6 & 35.93 & 60.60 & $\underline{65.23}$ & 58.81 & 51.27 & 61.78 & 62.52 \\
\hline & 0.2 & 0.2 & 0.2 & 0.5 & 56.58 & 73.58 & $\overline{65.68}$ & $72.63^{*}$ & 54.80 & 66.53 & $73.19^{\star}$ \\
\hline & 0.2 & 0.2 & 0.2 & 0.6 & 81.61 & 91.59 & 84.85 & 91.39* & 77.25 & 85.35 & $91.36^{\star}$ \\
\hline & 0.2 & 0.2 & 0.5 & 0.5 & 66.31 & $82.91^{*}$ & $82.69^{\star}$ & $\underline{83.13}$ & 65.20 & 70.88 & 76.72 \\
\hline & 0.3 & 0.3 & 0.6 & 0.6 & 61.23 & $78.91^{\star}$ & 79.02 & $78.22^{\star}$ & 61.16 & 68.11 & 73.05 \\
\hline & 0.2 & 0.5 & 0.5 & 0.5 & 49.76 & 68.86 & 58.05 & 69.61 & $72.03^{*}$ & 72.81 & 69.52 \\
\hline \multirow{17}{*}{30} & 0.1 & 0.2 & 0.3 & 0.4 & 67.59 & 87.07 & 90.16 & 85.52 & 82.83 & 88.56 & 87.59 \\
\hline & 0.2 & 0.3 & 0.4 & 0.5 & 56.83 & 79.83 & $\underline{83.66}$ & 77.41 & 73.67 & 80.26 & 81.01 \\
\hline & 0.1 & 0.1 & 0.1 & 0.4 & 86.24 & $93.85^{\star}$ & 89.42 & $\underline{94.71}$ & 83.97 & 89.85 & $94.21^{\star}$ \\
\hline & 0.2 & 0.2 & 0.2 & 0.5 & 75.36 & $87.41^{*}$ & 79.92 & $87.13^{*}$ & 71.08 & 80.49 & $\underline{87.44}$ \\
\hline & 0.1 & 0.1 & 0.4 & 0.4 & 92.83 & 97.77* & $97.24^{*}$ & $\underline{97.87}$ & 91.42 & 93.21 & 95.85 \\
\hline & 0.2 & 0.2 & 0.5 & 0.5 & 85.01 & $94.18^{\star}$ & 93.55* & $\underline{94.25}$ & 83.55 & 85.96 & 90.82 \\
\hline & 0.1 & 0.2 & 0.2 & 0.4 & 66.21 & 84.36 & 85.49 & 82.20 & 80.41 & $\underline{87.38}$ & $86.98^{\star}$ \\
\hline & 0.1 & 0.2 & 0.4 & 0.4 & 80.34 & $92.98^{\star}$ & $\underline{93.70}$ & 92.52 & 89.90 & 92.17 & 91.67 \\
\hline & 0.1 & 0.4 & 0.4 & 0.4 & 79.89 & 91.93 & 79.38 & 91.81 & $\underline{93.18}$ & 93.17 & 91.76 \\
\hline & 0.2 & 0.5 & 0.5 & 0.5 & 70.43 & 85.36 & 74.11 & 84.84 & $\underline{89.80}$ & 87.59 & 85.47 \\
\hline & 0.1 & 0.1 & 0.2 & 0.4 & 80.07 & $\underline{92.34}$ & 82.82 & $92.08^{*}$ & 82.07 & 88.65 & $91.98^{\star}$ \\
\hline & 0.1 & 0.1 & 0.3 & 0.4 & 84.47 & $\overline{94.70^{*}}$ & $\underline{95.40}$ & $94.51^{*}$ & 89.68 & 89.81 & 92.82 \\
\hline & 0.1 & 0.3 & 0.3 & 0.4 & 64.19 & 83.91 & 82.14 & 82.20 & 83.87 & $\underline{87.97}$ & 85.82 \\
\hline & 0.1 & 0.3 & 0.4 & 0.4 & 74.71 & 90.37 & 87.82 & 89.68 & 89.89 & 91.83 & 90.33 \\
\hline & 0.2 & 0.6 & 0.6 & 0.6 & 93.37 & $97.85^{\star}$ & 91.96 & $97.90^{*}$ & $\underline{98.78}$ & $98.30^{*}$ & 97.55 \\
\hline & 0.3 & 0.3 & 0.4 & 0.7 & 71.51 & $87.39^{\star}$ & 85.42 & $87.18^{*}$ & 70.86 & 81.09 & $\underline{87.92}$ \\
\hline & 0.3 & 0.3 & 0.6 & 0.7 & 78.49 & $91.82^{*}$ & 92.66 & 91.00 & 76.71 & 84.68 & 89.67 \\
\hline
\end{tabular}

8.2 An example of sample size determination in a dose-response study

When we calculate the required number of subjects for a dose-response study, we first estimate the 
response rate of each dose group and then select a testing procedure based on an expected response pattern and the primary hypothesis. The estimation of response rates is usually based on assumptions or earlier study results. Thus the assumed response rates for the planned study are not restricted to just one tuple of response rates. It is better to consider several plausible tuples of response rates. Furthermore, the power of the test depends on the response pattern. Therefore, it is advisable to calculate sample size for each test discussed in previous sections for several cases of response pattern, and choose the most efficient and also robust test.

Let us consider an example, see Minaguchi et al.(1996). Suppose we plan a dose-response study where four doses of a drug are compared based on a quantal response. The doses are equi-spaced on a logarithmic scale, so $D_{1}=D, D_{2}=2 D, D_{3}=4 \mathrm{D}$ and $D_{4}=8 \mathrm{D}$. The response rates of the lowest dose group and the maximum dose group were estimated to be $\mathrm{p}_{1}=0.20$ and $\mathrm{p}_{4}=0.45$ based on previous experiments. $\mathrm{D}_{3}$ is assumed to give almost the maximum effect, so that $\mathrm{p}_{3}$ is assumed to be 0.40 or $0.45 . \mathrm{D}_{2}$ is assumed to be less effective than $D_{3}$. Based on these considerations, the power of each test was calculated for samples of size $n_{1}=n_{2}=n_{4}=n_{3}=25,30,35,40$ and 45 and for seven combinations of $p_{2}$ and $p_{4}$;

case $1:(0.20,0.20,0.20,0.45), \quad$ case $2:(0.20,0.20,0.40,0.45), \quad$ case $3:(0.20,0.20,0.45,0.45)$,

case $4:(0.20,0.25,0.40,0.45), \quad$ case $5:(0.20,0.25,0.45,0.45), \quad$ case $6:(0.20,0.30,0.40,0.45)$,

case $7:(0.20,0.40,0.40,0.45)$.

The chi-bar square test and Cochran-Armitage test were comparable for case 2, 3 and 5. The chi-bar square test was preferred to the Cochran-Armitage test for case 1 and 7, and vise-versa for case 4 and 6 . From these results it was recommended to use the chi-bar square test and to enroll 40 subjects to each dose group. Because the chi-bar square test give the minimum value among seven tests of the maximum sample size among four combinations.

\subsection{Sample sizes for several cases}

It is very valuable to know the sample size for typical cases. From the results of power comparisons, it may be a good strategy to use the chi-bar square test or Williams test depending on response rates of intermediate dose levels. Table 5 gives sample sizes of required to assure $80 \%$ or $90 \%$ of powers for these two test of one-sided $5 \%$ level significance for experiments of three doses and $\mathrm{p}_{4}-\mathrm{p}_{1}$ $=0.20$ and $p_{4}-p_{1}=0.30$. The sample sizes were calculated by the method described in section 7.3.

Table 5a. Sample sizes of chi-bar square and Williams type tests in case of three dose with $5 \%$ level of significance and $80 \%$ of power.

\begin{tabular}{|c|c|c|c|c|c|c|c|c|c|c|c|c|c|c|}
\hline $\mathbf{p}_{1}$ & $\mathbf{p}_{2}$ & $\mathbf{p}_{3}$ & $\bar{x}^{2}$ & $\mathrm{Z}$ & $\mathrm{p}_{1}$ & $\mathbf{p}_{2}$ & $\mathrm{p}_{3}$ & $\bar{x}^{2}$ & $\mathrm{Z}$ & $\mathrm{p}_{1}$ & $\mathrm{p}_{2}$ & $\mathrm{p}_{3}$ & $\bar{x}^{2}$ & $\mathrm{Z}$ \\
\hline 10 & 10 & 30 & 40 & 46 & 10 & 20 & 30 & 51 & 48 & 10 & 30 & 30 & 43 & 42 \\
\hline 20 & 20 & 40 & 55 & 64 & 20 & 30 & 40 & 69 & 64 & 20 & 40 & 40 & 57 & 55 \\
\hline 30 & 30 & 50 & 64 & 77 & 30 & 40 & 50 & 79 & 76 & 30 & 50 & 50 & 65 & 62 \\
\hline 40 & 40 & 60 & 67 & 79 & 40 & 50 & 60 & 83 & 78 & 40 & 60 & 60 & 67 & 64 \\
\hline 50 & 50 & 70 & 65 & 78 & 50 & 60 & 70 & 79 & 77 & 50 & 70 & 70 & 64 & 62 \\
\hline 60 & 60 & 80 & 57 & 70 & 60 & 70 & 80 & 69 & 66 & 60 & 80 & 80 & 55 & 53 \\
\hline 70 & 70 & 90 & 43 & 55 & 70 & 80 & 90 & 51 & 49 & 70 & 90 & 90 & 40 & 38 \\
\hline 10 & 10 & 40 & 20 & 23 & 10 & 25 & 40 & 26 & 24 & 10 & 40 & 40 & 22 & 21 \\
\hline 20 & 20 & 50 & 26 & 31 & 20 & 35 & 50 & 33 & 31 & 20 & 50 & 50 & 27 & 27 \\
\hline 30 & 30 & 60 & 29 & 33 & 30 & 45 & 60 & 37 & 36 & 30 & 60 & 60 & 29 & 28 \\
\hline 40 & 40 & 70 & 29 & 37 & 40 & 55 & 70 & 37 & 36 & 40 & 70 & 70 & 29 & 28 \\
\hline 50 & 50 & 80 & 27 & 32 & 50 & 65 & 80 & 33 & 32 & 50 & 80 & 80 & 26 & 25 \\
\hline 60 & 60 & 90 & 22 & 27 & 60 & 75 & 90 & 26 & 25 & 60 & 90 & 90 & 20 & 20 \\
\hline
\end{tabular}


Validity and Applicability of Tests for Ordered Alternatives

Table 5b. Sample sizes of chi-bar square and Williams type tests in case of three dose with 5\% level of significance and $90 \%$ of power.

\begin{tabular}{|c|c|c|c|c|c|c|c|c|c|c|c|c|c|c|}
\hline$\underline{p}_{1}$ & $\mathrm{p}_{2}$ & $\mathrm{p}_{3}$ & $\bar{x}^{2}$ & $\mathrm{z}$ & $\mathrm{p}_{1}$ & $\mathrm{p}_{2}$ & $\mathrm{p}_{3}$ & $\bar{x}^{-2}$ & $\mathrm{z}$ & $\mathrm{p}_{1}$ & $\mathrm{p}_{2}$ & $\mathrm{p}_{3}$ & $\bar{x}^{2}$ & $\overline{\mathrm{Z}}$ \\
\hline 10 & 10 & 30 & 54 & 63 & 10 & 20 & 30 & 70 & 66 & 10 & 30 & 30 & 58 & 56 \\
\hline 20 & 20 & 40 & 74 & 89 & 20 & 30 & 40 & 95 & 90 & 20 & 40 & 40 & 77 & 74 \\
\hline 30 & 30 & 50 & 87 & 103 & 30 & 40 & 50 & 110 & 105 & 30 & 50 & 50 & 88 & 87 \\
\hline 40 & 40 & 60 & 91 & 109 & 40 & 50 & 60 & 114 & 108 & 40 & 60 & 60 & 91 & 89 \\
\hline 50 & 50 & 70 & 88 & 108 & 50 & 60 & 70 & 110 & 106 & 50 & 70 & 70 & 87 & 84 \\
\hline 60 & 60 & 80 & 77 & 97 & 60 & 70 & 80 & 95 & 90 & 60 & 80 & 80 & 74 & 73 \\
\hline 70 & 70 & 90 & 58 & 73 & 70 & 80 & 90 & 70 & 68 & 70 & 90 & 90 & 54 & 53 \\
\hline 10 & 10 & 40 & 28 & 31 & 10 & 25 & 40 & 35 & 33 & 10 & 40 & 40 & 29 & 28 \\
\hline 20 & 20 & 50 & 35 & 42 & 20 & 35 & 50 & 45 & 42 & 20 & 50 & 50 & 36 & 36 \\
\hline 30 & 30 & 60 & 39 & 48 & 30 & 45 & 60 & 50 & 48 & 30 & 60 & 60 & 39 & 38 \\
\hline 40 & 40 & 70 & 39 & 49 & 40 & 55 & 70 & 50 & 48 & 40 & 70 & 70 & 39 & 38 \\
\hline 50 & 50 & 80 & 36 & 43 & 50 & 65 & 80 & 45 & 43 & 50 & 80 & 80 & 35 & 35 \\
\hline 60 & 60 & 90 & 29 & 37 & 60 & 75 & 90 & 35 & 35 & 60 & 90 & 90 & 28 & 26 \\
\hline
\end{tabular}

\section{Acknowledgment}

The author wishes to express his appreciation to the editor and referees for their valuable comments.

\section{References}

Armitage,P.(1955) Tests for linear trends in proportions and frequencies. Biometrics 11, 375-386.

Barlow,R.E., Bartholomew,D.J., Bremner,J.M. and Brunk, H.D.(1972) Statistical Inference under order Restriction. Wiley.New York.

Bartholomew,D.J.(1959a) A test of homogeneity for order alternatives. Biometrika 46, 36-48.

Bartholomew,D.J.(1959b) A test of homogeneity for ordered alternatives II. Biometrika 46, 323-335.

Berenson, M.L.(1982) A comparison of several $\mathrm{k}$ sample tests for ordered alternatives in completely randomized designs. Psychometrika 47, 265-280.

Budde, M. and Bauer, P.(1989) Multiple test procedures in clinical dose finding studies. Journal of American Statistical Association 84, 792-796.

Chacko,V.J.(1963) Testing homogeneity against ordered alternatives. Annals of Mathematical Statistics 29, 945-956.

Cochran,W.G.(1955) Some methods for strengthening the common $\mathrm{X}^{2}$ tests. Biometrics 11, 417-451.

Cox,D.R.(1958) The regression analysis of binary sequences. Journal of Royal Statistical Society. B20, 215-242 (with discussion).

Dunnett,C.W.(1955) A multiple comparison procedure for comparing several treatments with a control. Journal of American Statistical Association 50, 1096-1121.

Dunnett,C.W.(1964) New tables for multiple comparisons with control. Biometrics 20, 482-491.

Hirotsu, C., Kuriki, T. and Hayter, A. J. (1992)Multiple comparison procedures based on the maximal component of cumulative chi-squared statistic. Biometrika 79,381-392.

Jonckheere,A.R.(1954) A distribution-free k-sample test against ordered alternatives. Biometrika 41, 133-145.

Kuriki,T., Hirotsu,C. and Hayter,A.J.(1989) The multiple comparison procedure based on the maximal component of the cumulative chi-square statistic - Calculation of significance probability and its application to comparison of dose levels-. Japanese Journal of Applied statistics 18, 129-141 (in Japanese).

Marcus,R.(1976) The powers of some tests of the equality of normal means against an ordered alternative. Biometrika 63, 177-183. 
Minaguchi, H., Mizuno, M., Tanizawa, O., Sugimoto, O., Nagata, Y. and Tango, T. (1996) Dose-Finding study of a sustained release preparation of Bucerelin acetate micro-particles on patients with leyomyoma. Sanfujinka no Sekai 48, 221-248. (in Japanese)

Ruberg, S.J.(1989) Contrasts for identifying the minimum effective dose. Journal of American Statistical Association 84, 816-822.

Shirley, E.A.C.(1979) The comparison of treatment with control group means in toxicological studies. Applied Statistics 28, 144-151.

Shirley, E.A.C.(1985) The values of specialized tests in studies where ordered group means are expected. Statistics in Medicine 4, 489-496.

Shorack,G.R.(1967) Testing against ordered alternatives in model I analysis of variance; Normal theory and monparametric. Annals of Mathematical Statistics 1740-1752.

Taron,R.E. and Gart,J.J.(1980) On the robustness of combined tests for trends in proportions. Journal of American Society 75, 110-116.

Terpstra,T. J. (1952) The asymptotic normality and consistency of Kendall's test against trend,when ties are present in one ranking. Indagations Math., 14,327-333.

Williams,D.A.(1971) A test for differences between treatment means when several dose levels are compared with zero dose control. Biometrics 27, 103-117.

Williams,D.A.(1972) The comparison of several dose levels with a zero dose control. Biometrics 28 , 519-531.

(Received October 1997; Revised December 1998) 